\title{
1 The subtle origins of surface-warming hiatuses
}

2

Christopher Hedemann ${ }^{1,2 *}$, Thorsten Mauritsen ${ }^{1}$, Johann Jungclaus ${ }^{1}$ and Jochem Marotzke $^{1}$

${ }^{1}$ Max-Planck-Institut für Meteorologie, Bundestraße 53, 20146 Hamburg, Germany

${ }^{2}$ International Max Planck Research School on Earth System Modelling, MaxPlanck-Institut für Meteorologie, Bundestraße 53, 20146 Hamburg, Germany

*email: christopher.hedemann@mpimet.mpg.de

During the first decade of the $21^{\text {st }}$ Century, the Earth's surface warmed more slowly than climate models simulated ${ }^{1}$. This surface-warming hiatus is attributed by some studies to model errors in external forcing ${ }^{2-4}$, while others point to heat rearrangements in the ocean ${ }^{5-10}$ caused by internal variability, the timing of which cannot be predicted by the models ${ }^{1}$. However, observational analyses disagree about which ocean region is responsible ${ }^{11-16}$. Here we show that the hiatus could also have been caused by internal variability in the top-ofatmosphere energy imbalance. Energy budgeting for the ocean surface layer over a 100-member historical ensemble reveals that hiatuses are caused by energy-flux deviations as small as $0.08 \mathrm{Wm}^{-2}$, which can originate at the top of the atmosphere, in the ocean, or both. Budgeting with existing observations cannot constrain the origin of the recent hiatus, because the uncertainty in observations dwarfs the small flux deviations that could cause a hiatus. The sensitivity of these flux deviations to the observational dataset and to energy budget choices helps explain why previous studies conflict, and suggests that the origin of the recent hiatus may never be identified.

The surface temperature of the Earth warmed more slowly over the period 1998-2012 than could be expected by examining either most model projections or the long-term warming trend ${ }^{1}$. Even though some studies now attribute the deviation from the longterm trend to observational biases ${ }^{17,18}$, the gap between observations and models persists. The observed trend deviated by as much as $-0.17^{\circ} \mathrm{C}$ per decade from the CMIP5 (Coupled Model Intercomparison Project Phase 5; ref. 19) ensemble mean projection $^{1}$ - a gap two to four times the observed trend. The hiatus therefore continues to challenge climate science. 
Many studies propose that heat was drawn down from the surface into deeper ocean layers by quasi-random decadal fluctuations known as internal variability. The trouble with this proposition is that most major ocean regions - the Pacific ${ }^{12,14}$, the Indian Ocean $^{15}$, the Atlantic ${ }^{10}$, the Atlantic and the Southern Ocean ${ }^{13}$, and other combinations of basins $^{5-7,11,16}$ - have been named individually responsible for the heat uptake.

Here we explain these conflicting results and point to alternative interpretations. We develop a surface energy budget, which we apply to hiatuses in a 100-member historical ensemble ('the large ensemble'), generated with the coupled climate model MPI-ESM1.1 (Methods; ref. 20). Using the surface energy budget, we quantify how much deviation in energy flux occurs during a hiatus. For each hiatus in the ensemble, we then determine its origin by quantifying energy contributions to the surface from the ocean and from the top-of-atmosphere (TOA) radiative imbalance (Supplementary Fig. 1). Finally, we use the energy budget to compare interpretations of the recent hiatus in existing observations ${ }^{9,21-23}$.

We define hiatuses in the large ensemble as any 15-year period where the GMST trend deviates by at least $-0.17^{\circ} \mathrm{C}$ per decade from the ensemble mean. This definition is consistent with the gap between models and observations over the period 1998-2012 (Fig. 1), as described in the Intergovernmental Panel on Climate Change Assessment Report 5 (ref. 1). Deviations in each ensemble member from the largeensemble mean represent internal variability, which can be cleanly separated from the forced component (the ensemble mean) due to the ensemble's unprecedented size. There are hundreds of such hiatuses (364, or $2.4 \%$ of all 15,200 trends) - subject to historical forcing but due entirely to internal variability - distributed across all time periods in the ensemble (Fig. 1).

The origin of each hiatus can be deduced from energy budgeting for the ocean's surface layer (Supplementary Fig. 1), which dominates the thermal capacity of the Earth's surface and therefore mediates the decadal GMST response to flux perturbations. We consider two main flux components acting on the ocean surface layer over decadal timescales: the TOA component from above and the ocean component from below (Fig. 2a). The TOA component is the top-of-atmosphere radiative flux imbalance minus atmospheric heat uptake. The ocean component is the total heat-content change below the ocean surface layer, defined at 100m depth. Both 
components are converted to ensemble anomalies (to isolate the internal variability component) from values filtered over a 15-year sliding window (see Methods) and warm the surface layer when positive.

The budget is constructed this way for two reasons. Firstly, the chosen boundary fluxes (Fig. 2a) close the surface energy budget: the sum of the TOA component and ocean component highly correlates with heat-content changes within the ocean surface layer $\left(r^{2}=0.97\right.$, slope $=1.00$; Supplementary Fig. 2). Other flux components (Fig. 2a) are excluded because they are small, are connected with known energy leakages, and because they do not improve budget closure (Methods; Supplementary Fig. 2b). The TOA imbalance and ocean heat uptake dominate decadal internal variability in the global energy budget of other CMIP5 models as well ${ }^{27}$. Secondly, the ocean surface layer is defined at 100m (as in refs. 24-26), because around this depth the flux-divergence anomaly for a hiatus reaches a maximum (Fig. 2b) and is therefore the most conservative choice for our analysis. Choosing a surface depth beyond $100 \mathrm{~m}$ further exceeds the globally averaged mixed layer, and so the correlation between the energy budget and GMST trends sharply decays (Fig. 2b).

The energy budget allows us to determine the magnitude of flux anomalies associated with each hiatus. From the slope of the regression between surface-layer fluxdivergence and GMST trends, we find that the expected flux-divergence anomaly for a hiatus ( $\mathrm{a}-0.17{ }^{\circ} \mathrm{C}$ per decade anomaly) is merely $-0.082 \mathrm{Wm}^{-2}$ (Methods). This corresponds to an average cooling over the ocean's top $100 \mathrm{~m}$ of only $-0.10{ }^{\circ} \mathrm{C}$ per decade (Methods) but the effects of that cooling are amplified at the land surface ${ }^{28}$. Hiatuses caused only by the ocean tend to cool the land surface more effectively, which means they generally require a lower flux-divergence anomaly than other hiatuses to achieve the same cooling. Variation in the ratio of land to ocean surfacecooling leads to variation around the expected flux-divergence anomaly: an interval of $-0.082 \pm 0.038 \mathrm{Wm}^{-2}$ covers the 5-95\% range for all hiatuses. These results suggest that the total combined anomaly in TOA fluxes and ocean heat uptake that caused the gap between observations and models during the hiatus could be on the order of 0.1 $\mathrm{Wm}^{-2}$. Defining hiatuses as equal to the observed 1998-2012 anomaly from the longterm observed trend (an anomaly of $0.04-0.07^{\circ} \mathrm{C}$ per decade) would reduce the threshold to just $0.02-0.03 \mathrm{Wm}^{-2}$. 
99 Across the large ensemble, the $0.082 \mathrm{Wm}^{-2}$ threshold in energy flux is frequently

100 exceeded by anomalous heat-content changes in all major ocean basins, especially in

101 the Atlantic, Pacific and Southern Oceans (Fig. 3b). However, these heat-content

102 changes are dominated by interbasin heat exchange, which does not contribute to the

103 surface-layer flux-divergence. In each major basin, the variations in heat content

104 below the surface layer cannot predict trends in GMST (Fig. 3a), and indeed would

105 falsely predict many more hiatuses than actually occur.

106 Even the global ocean heat uptake below $100 \mathrm{~m}$ correlates poorly with GMST trends

107 (Fig 3a). The TOA component tends to oppose the ocean component's contribution to

108 the energy budget, as demonstrated by the negative correlation in Figure 3c. The flux-

109 divergence anomaly, which has less than half the variability of either the TOA or

110 ocean component alone (Fig. 3b), is the only reliable predictor of GMST trends (Fig.

$1113 a)$.

112 The role of the TOA and the ocean in each hiatus can be determined by comparing

113 their relative contributions to the flux-divergence anomaly. For hiatuses in the large

114 historical ensemble, the negative (cooling) anomaly is caused entirely by the TOA in

$11512 \%$ of cases and by the ocean in $24 \%$. In the remainder (64\%), the negative anomaly

116 is caused by the TOA and ocean acting together (bottom left quadrant of Fig. 3c).

117 TOA variability is therefore involved in $76 \%$ of all hiatuses.

118 Applying a similar analysis to observations should reveal the energetic origin of the

119 gap between models and observations during the recent hiatus (Supplementary Fig.

120 1). We convert two observation-based estimates of fluxes over 2000-2010 to

121 anomalies by subtracting the mean energy budget of the large ensemble for the same

122 period (Methods). These anomalies include both the effect of internal variability and

123 any potential effects of forcing differences between model and observations.

124 Choosing 2000-2010 means that we do not cover the full hiatus period (1998-2012)

125 and that the corresponding gap in GMST trend between models and observations is

126 reduced, because the warming rate increased after 2000 (ref. 18). However, this

127 choice allows us to construct temporally consistent energy budgets from multiple

128 sources and to take advantage of the improved quality of observations after 2000.

129 Although the budgets do not cover the full hiatus period, they do illustrate how

130 observational uncertainty affects interpretations of the hiatus. The first budget uses

131 WOA ocean observations ${ }^{22}$ and a recent estimate of TOA fluxes based on the CERES 
132 satellite data product, Argo floats and AMIP simulations ${ }^{21}$. This first budget suggests

133 that the hiatus was caused purely by the reduced influx of energy at the TOA (orange

134 dot, Fig. 3c). The second budget, based on ocean reanalysis data from ORAS4 (refs.

$1359,23)$, suggests the hiatus was caused purely by increased heat uptake in the ocean

136 (green dot, Fig. 3c). The anomalies diagnosed from an ocean model forced with the

137 exceptional Pacific trade winds observed during the hiatus ${ }^{12}$ likewise suggest an

138 ocean origin (purple dot, Fig. 3c).

139 From our analysis of observational estimates, we are unable to exclude the TOA

140 anomaly as a possible cause of the recent hiatus. Referencing the observations to an

141 alternative energy budget (rather than that of the large ensemble) could shift the

142 absolute position of the green and orange crosses in Figure 3c. However, their relative

143 distance from one another and the size of their error bars would not change.

144 Interpretations of the hiatus are also sensitive to the energy budgeting method used,

145 and this may reveal why the results of previous studies conflict. For example, the

146 hiatus has been explained as the result of heat being transferred from the surface

147 ocean to the layers immediately below it, in the upper 300-350m (ref. 14, 16).

148 However, an energy budget that only accounts for heat exchange between the top

$149100 \mathrm{~m}$ and depths up to $300-350 \mathrm{~m}$ correlates poorly with GMST trends in the large

150 ensemble $\left(r^{2}=0.08\right.$, Supplementary Fig. 4). A poor correlation also results when we

151 exclude heat-content changes below the upper $700 \mathrm{~m}\left(\mathrm{r}^{2}=0.14\right.$, Supplementary Fig. 4;

152 see ref. 15) and the upper $2000 \mathrm{~m}$ of ocean $\left(\mathrm{r}^{2}=0.36\right.$, Supplementary Fig. 4; see ref.

153 13). Heat-content changes up to as much as $4000 \mathrm{~m}$ may be important for decadal

154 internal variability (Supplementary Fig. 4), despite claims to the contrary ${ }^{16}$.

155 Furthermore, the pattern of surface-layer cooling overlying a warming trend may be

156 common during ocean hiatuses, but it also occurs in around half of hiatuses caused

157 purely by the TOA (Supplementary Fig. 5). During these TOA hiatuses, the

158 subsurface warming is caused by heat transfer from deeper layers. Energy budgets

159 that do not consider uptake across the whole ocean depth may therefore misrepresent

160 crucial energy fluxes and misdiagnose the hiatus.

161 The hiatus may also be misdiagnosed by misrepresenting the surface layer in energy

162 budgeting. For example, the surface layer has been defined at $300 \mathrm{~m}$ ocean depth or

163 more ${ }^{5,6,8-10,13}$. We perform energy budgeting in the large ensemble with a surface 
layer that extends to $300 \mathrm{~m}$ instead of $100 \mathrm{~m}$ and find that the flux-divergence correlates comparatively poorly with GMST trends $\left(r^{2}=0.33\right.$ for $300 m$, Fig. $\left.2 b\right)$.

We conclude that the TOA may have been a source of significant internal variability during the hiatus. Our conclusions are not an artefact of model-generated TOA variability $^{29}$ - the large ensemble produces TOA variability that is similar to that in the observational record (Supplementary Fig. 6). Rather, our conclusions are based on a simple yet robust principle, namely that the Earth's surface layer has a small heat capacity. The surface temperature can therefore be influenced by small variations in the large yet mutually compensating fluxes that make up this layer's energy budget. Comparing the small variability in the TOA imbalance with the total TOA imbalance under global warming ${ }^{26,30}$ obscures the significance of these small variations for the hiatus.

Other observational studies associate the hiatus with heat-flux anomalies that range from $0.21 \mathrm{Wm}^{-2}$ (ref. 30) to $0.50 \mathrm{Wm}^{-2}$ (ref. 11). But when we perform energy budgeting for the surface layer in the large ensemble, we find that anomalies closer to $0.08 \mathrm{Wm}^{-2}$ can account for hiatuses as large as $0.17^{\circ} \mathrm{C}$ per decade, and $0.02-0.03$ $\mathrm{Wm}^{-2}$ for a hiatus equal to the 1998-2012 anomaly from the observed long-term trend. Because the flux-divergence anomaly is so small, ascribing the origin of the recent hiatus to the TOA or ocean requires that each of their contributions to the anomaly are known with considerable accuracy. However, the uncertainty in TOA imbalance from satellite measurements is two orders of magnitude larger $\left(\sim 8 \mathrm{Wm}^{-2}\right.$; ref. 31) than the anomaly we calculate. Satellite data are commonly anchored with ocean heat-content measurements, but the uncertainty range in TOA imbalance during the $2000 \mathrm{~s}$ still remains around $0.56 \mathrm{Wm}^{-2}$ (ref. 21), and even for the most recent estimate based on improved ocean observations over 2005-2015, the range is 0.2 $\mathrm{Wm}^{-2}$ (ref. 32).

This is the true dilemma at the heart of the hiatus debate: the variability in ocean heat content alone has no power to explain the hiatus, and the measure that can - the surface-layer flux-divergence - is dwarfed by observational uncertainty. While there are attempts to fill the gaps in observations with ocean reanalyses like ORAS4 (refs. $9,23)$, the resulting data are of questionable integrity during the hiatus ${ }^{14,21}$ and, as we show, disagree with the budget based on $\mathrm{CERES}^{21}$ and $\mathrm{WOA}^{22}$. Even if these disagreements could be reconciled, the process of anchoring satellite observations 
197 with ocean heat uptake makes the contributions from TOA and ocean difficult to

198 disentangle, because their absolute difference is unknown. Therefore, unless the

199 uncertainty of observational estimates can be considerably reduced, the true origin of

200 the recent hiatus may never be determined. 


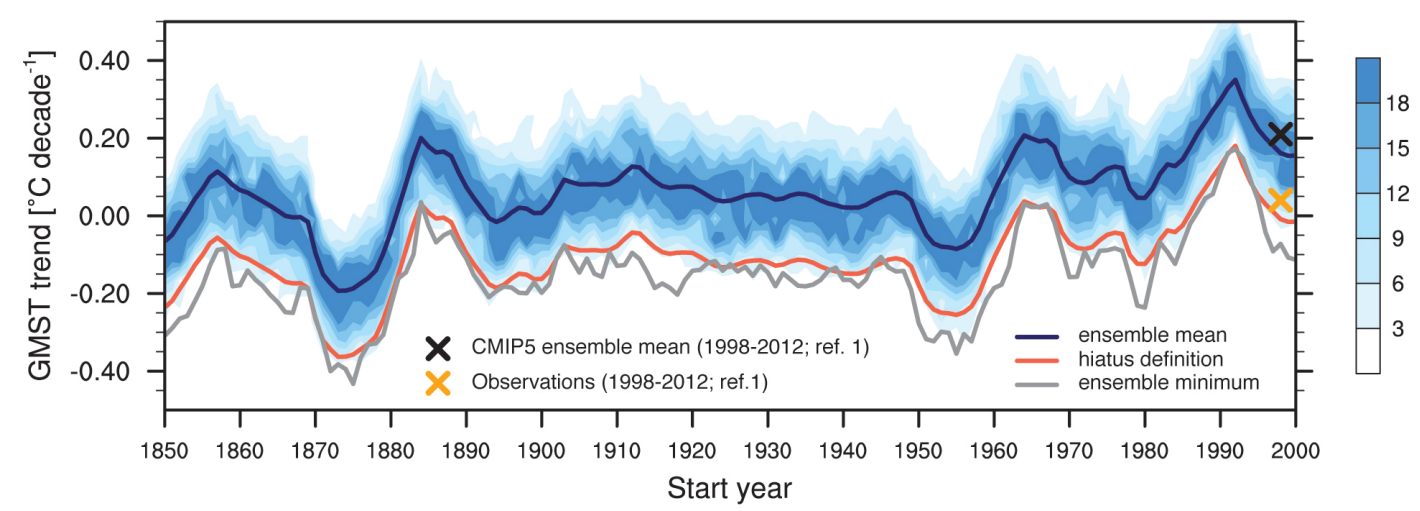

Figure 1 | Distribution of 15-year trends in global mean surface temperature (GMST) in the 100member ensemble. The coupled climate model MPI-ESM1.1 is forced with CMIP5-prescribed historical forcing from 1850 until 2005, and extended until 2015 with the RCP4.5 scenario (see Methods). When the red line lies above the grey line, at least one ensemble member is experiencing a hiatus, defined as a deviation of more than $0.17^{\circ} \mathrm{C}$ per decade below the ensemble mean. This deviation is the same as the gap between the CMIP5 ensemble mean (black cross) and the observed (yellow cross) GMST trends for the period 1998-2012. Contours represent the number of ensemble 211 members in bins of $0.05^{\circ} \mathrm{C}$ per decade.

a

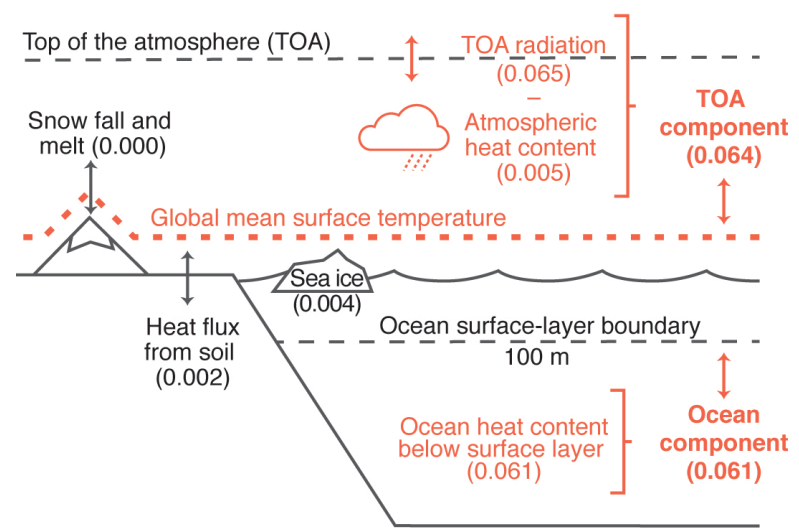

b

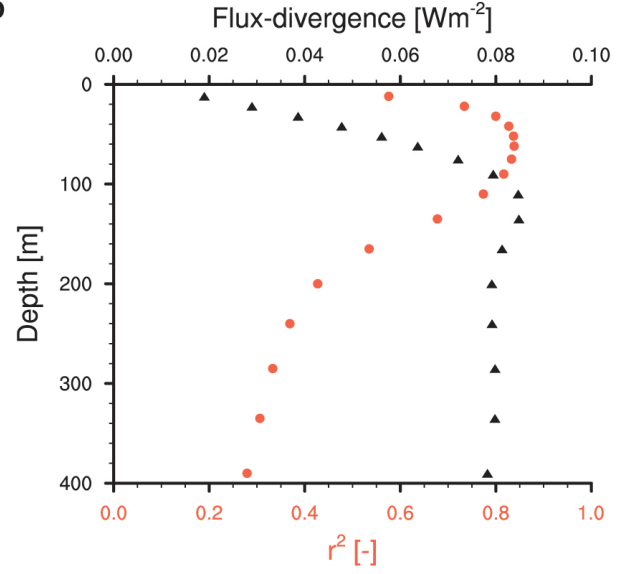

Figure 2 | Surface energy budgets. a, The surface energy budget in the large ensemble. Red colouring indicates the global mean surface temperature (GMST) and the components included in the surfacelayer flux-divergence. The smaller flux components in black are excluded because they do not improve budget closure or the relationship with GMST trends. Numbers in brackets represent the variability of each heat flux $\left(\mathrm{Wm}^{-2}\right)$, given as the root-mean-square of 15-year ensemble anomalies. $\mathbf{b}$, Results from surface budgets determined by increasingly deeper definitions of the ocean surface layer. For each depth, a linear regression is performed for GMST trends against the surface-layer flux-divergence (both as 15 -year ensemble anomalies). Shown in black (top axis) is the expected deviation in flux-divergence required to cause a hiatus, calculated from the regression slope. Shown in red (bottom axis) is the correlation $\left(\mathrm{r}^{2}\right)$ of each regression. The correlation rapidly deteriorates for definitions of the surface 224 layer below $100 \mathrm{~m}$. 
a

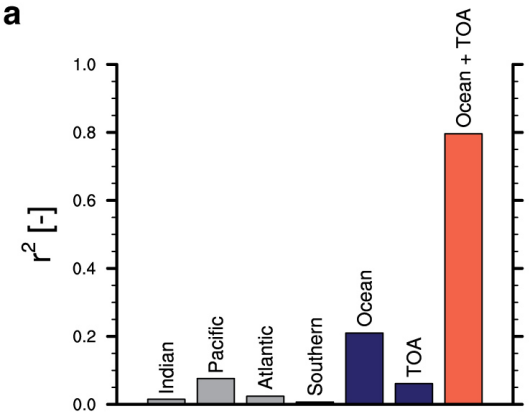

b

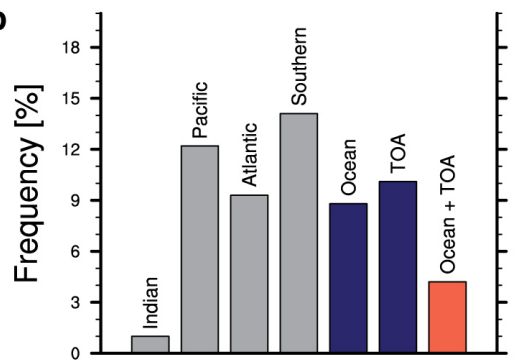

C

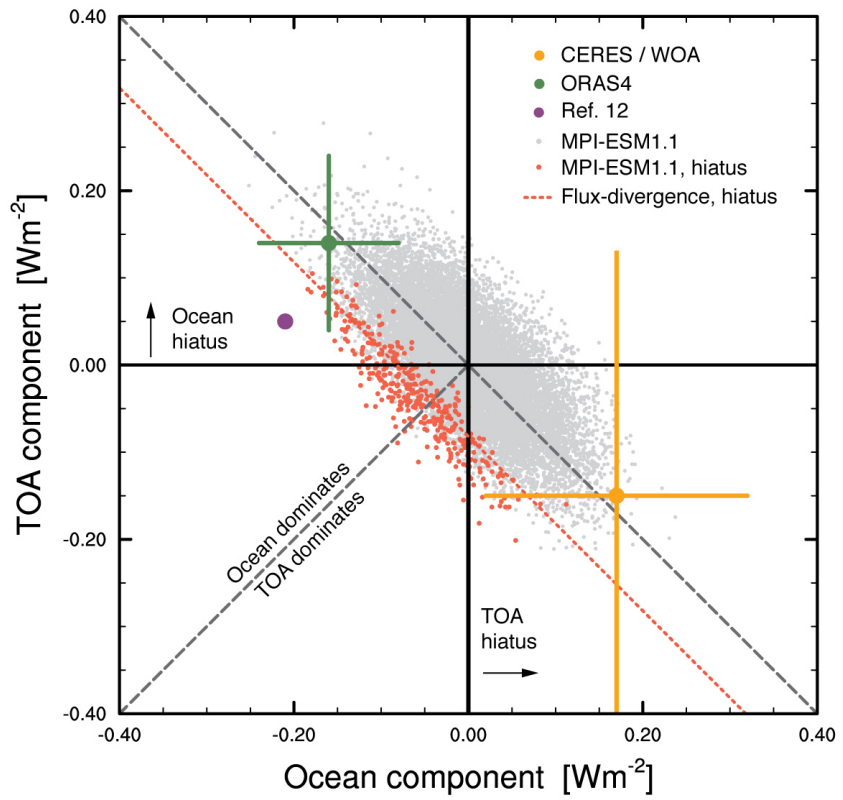

Figure 3 | Hiatuses and their origins in models and observations. a, Correlation between global mean surface temperature (GMST) trends and heat fluxes in the large ensemble (as 15-year ensemble anomalies). b, Frequency with which each component exceeds the expected threshold for a hiatus ($\left.0.082 \mathrm{Wm}^{-2}\right)$. In $\mathbf{a}$ and $\mathbf{b}$, grey bars represent changes in ocean heat content below the ocean surface layer $(100 \mathrm{~m})$ by basin, blue bars represent the ocean and TOA components, and the red bar is the surface-layer flux-divergence (TOA + ocean components). c, Contributions to hiatuses from TOA and ocean components. Positive values indicate fluxes that warm the surface. Small red dots represent hiatuses in the large ensemble and small grey dots represent all other trends; the red dotted line is a flux-divergence of $-0.082 \mathrm{Wm}^{-2}$. Observational estimates and their 1-sigma error bars are compiled from multiple sources that rely either on $\mathrm{CERES}^{21}$ and WOA data ${ }^{22}$ (large orange dot) or ORAS4 data $^{9,23}$ (large green dot), shown as anomalies from the large-ensemble mean over the $2000 \mathrm{~s}(-0.66$ $\mathrm{Wm}^{-2}$ for the ocean and $+0.77 \mathrm{Wm}^{-2}$ for the TOA component). The large purple dot represents results from an ocean model forced with reanalysis-based winds as reported in ref. 12, converted to mean fluxes over 15 years.

\section{References}

1. Flato, G. et al. in Climate Change 2013: The Physical Science Basis. (eds. Stocker, T. F. et al.) 741-866 (Cambridge University Press, 2013).

2. Solomon, S. et al. The persistently variable 'background' stratospheric aerosol layer and global climate change. Science 333, 866-870 (2011).

3. Santer, B. D. et al. Volcanic contribution to decadal changes in tropospheric temperature. Nat. Geosci. 7, 185-189 (2014).

4. Kopp, G. \& Lean, J. L. A new, lower value of total solar irradiance: Evidence and climate significance. Geophys. Res. Lett. 38, 1-7 (2011).

5. Meehl, G. A., Arblaster, J. M., Fasullo, J. T., Hu, A. \& Trenberth, K. E. Modelbased evidence of deep-ocean heat uptake during surface-temperature hiatus periods. Nat. Clim. Chang. 1, 360-364 (2011).

6. Meehl, G. A., Hu, A., Arblaster, J. M., Fasullo, J. \& Trenberth, K. E. 
(2013).

7. Guemas, V., Doblas-Reyes, F. J., Andreu-Burillo, I. \& Asif, M. Retrospective prediction of the global warming slowdown in the past decade. Nat. Clim. Chang. 3, 649-653 (2013).

8. Watanabe, M. et al. Strengthening of ocean heat uptake efficiency associated with the recent climate hiatus. Geophys. Res. Lett. 40, 3175-3179 (2013).

9. Balmaseda, M. A., Trenberth, K. E. \& Källén, E. Distinctive climate signals in reanalysis of global ocean heat content. Geophys. Res. Lett. 40, 1754-1759 (2013).

10. Katsman, C. A. \& van Oldenborgh, G. J. Tracing the upper ocean's 'missing heat'. Geophys. Res. Lett. 38, (2011).

11. Drijfhout, S. S. et al. Surface warming hiatus caused by increased heat uptake across multiple ocean basins. Geophys. Res. Lett. 41, 7868-7874 (2014).

12. England, M. H. et al. Recent intensification of wind-driven circulation in the Pacific and the ongoing warming hiatus. Nat. Clim. Chang. 4, 222-227 (2014).

13. Chen, X. \& Tung, K.-K. Varying planetary heat sink led to global-warming slowdown and acceleration. Science 345, 897-903 (2014).

14. Nieves, V., Willis, J. K. \& Patzert, W. C. Recent hiatus caused by decadal shift in Indo-Pacific heating. Science 349, 532-535 (2015).

15. Lee, S.-K. et al. Pacific origin of the abrupt increase in Indian Ocean heat content during the warming hiatus. Nat. Geosci. 8, (2015).

16. Liu, W., Xie, S.-P. \& Lu, J. Tracking ocean heat uptake during the surface warming hiatus. Nat. Commun. 7:10926 (2016).

17. Cowtan, K. \& Way, R. G. Coverage bias in the HadCRUT4 temperature series and its impact on recent temperature trends. Q. J. R. Meteorol. Soc. 140, 19351944 (2014).

18. Karl, T. R. et al. Possible artifacts of data biases in the recent global surface warming hiatus. Science 348, 1469-72 (2015).

19. Taylor, K. E., Stouffer, R. J. \& Meehl, G. a. An overview of CMIP5 and the experiment design. Bull. Am. Meteorol. Soc. 93, 485-498 (2012).

20. Giorgetta, M. a. et al. Climate and carbon cycle changes from 1850 to 2100 in MPI-ESM simulations for the Coupled Model Intercomparison Project phase 5. J. Adv. Model. Earth Syst. 5, 572-597 (2013).

21. Smith, D. M. et al. Earth's energy imbalance since 1960 in observations and CMIP5 models. Geophys. Res. Lett. 42, 1205-1213 (2015).

22. Levitus, S. et al. World ocean heat content and thermosteric sea level change (0-2000 m), 1955-2010. Geophys. Res. Lett. 39, (2012).

23. Trenberth, K. E., Fasullo, J. T. \& Balmaseda, M. A. Earth's Energy Imbalance. J. Clim. 27, 3129-3144 (2014).

24. Baker, M. B. \& Roe, G. H. The shape of things to come: Why is climate change so predictable? J. Clim. 22, 4574-4589 (2009).

25. Geoffroy, O. et al. Transient climate response in a two-layer energy-balance 
model. Part I: Analytical solution and parameter calibration using CMIP5 AOGCM experiments. J. Clim. 26, 1841-1857 (2013).

26. Brown, P. T., Li, W., Li, L. \& Ming, Y. Top-of-atmosphere radiative contribution to unforced decadal global temperature variability in climate models. Geophys. Res. Lett. 41, 5175-5183 (2014).

27. Palmer, M. D. \& McNeall, D. J. Internal variability of Earth's energy budget simulated by CMIP5 climate models. Environ. Res. Lett. 9, 034016 (2014).

28. Byrne, P. B. \& O'Gorman, P. A. Land-ocean warming contrast over a wide range of climates: Convective quasi-equilibrium theory and idealized simulations. J. Clim. 26, 4000-4016 (2013).

29. Stephens, G. L. et al. The albedo of Earth. Rev. Geophys. 53, 141-163 (2015).

30. Trenberth, K. E. \& Fasullo, J. T. An apparent hiatus in global warming? Earth's Future. 1, 19-32 (2013).

31. Loeb, N. G. et al. Toward optimal closure of the Earth's top-of-atmosphere radiation budget. J. Clim. 22, 748-766 (2009).

32. Johnson, G. C., Lyman, J. M. \& Loeb, N. G. Improving estimates of Earth's energy imbalance. Nat. Clim. Chang. 6, 639-640 (2016).

\section{Corresponding author}

Correspondence to: Christopher Hedemann

\section{Acknowledgements}

This work is supported by the Max Planck Society for the Advancement of Science through the International Max Planck Research School on Earth System Modelling (IMPRS-ESM). J.J. acknowledges support from the European Union's Horizon 2020 research and innovation programme (grant agreement $n^{\circ}$ 633211). We thank Helmuth Haak for his technical assistance, Hao Zuo and Drew Peterson for providing the NEMO grid configuration, and Bjorn Stevens and Chao Li for their comments on the manuscript. We are indebted to Luis Kornblueh for producing the large historical ensemble and to Thomas Schulthess and the Swiss National Computing Centre (CSCS) for providing the necessary computational resources. Thanks also to Jürgen Kröger for producing the RCP4.5 extensions with the Deutsches Klimarechenzentrum (DKRZ) facilities.

\section{Author contributions}

C.H. and J.M. conceived the original idea for this study. C.H. developed the methodology and performed the analysis. All authors discussed the results. C.H. wrote the manuscript with input from J.M., T.M. and J.J.

\section{Competing financial interests}

The authors declare no competing financial interests. 


\section{Methods}

339 The large historical ensemble in this study was generated by the Max Planck Institute

340 Earth System Model version 1.1 (MPI-ESM1.1), an incremental improvement of the

341 coupled ocean-atmosphere general circulation model submitted to CMIP5 in the LR

342 configuration $^{20}$. The 100 ensemble members were generated under CMIP5 historical

343 forcing from 1850 until 2005, with extensions to 2015 under the RCP4.5 scenario $^{20}$.

344 The ensemble's internal variability of 15 -year GMST trends $\left(5-95 \%\right.$ range of $0.30{ }^{\circ} \mathrm{C}$

345 per decade) is slightly larger than an estimate for the CMIP5 ensemble (5-95\% range

346 of $0.26^{\circ} \mathrm{C}$ per decade; ref. 33 ).

347 GMST trends are calculated from the slope of an ordinary least-squares linear

348 regression over a 15-year sliding window, to be consistent with the hiatus as

349 described in ref. 1. Ensemble anomalies are then calculated at each time step:

$350 \quad X_{t, n}^{\prime}=X_{t, n}-\frac{1}{100} \sum_{n=1}^{100} X_{t, n}$, where $t$ is the time-step and $n$ is the ensemble member.

351 The composition of the energy budget is chosen to maximise the correlation of the

352 surface-layer flux-divergence with both GMST trends and changes in ocean surface-

353 layer heat content.

354 For the comparison with GMST trends (Supplementary Fig. 2) and most of this study,

355 any terms expressed as heat content (Joules) are converted to trend anomalies in the

356 same way as GMST, and then converted to units of $\mathrm{Wm}^{-2}$ over the total surface area

357 of the Earth. All energy fluxes that are output from the model as $\mathrm{Wm}^{-2}$ are first time-

358 integrated and then treated the same as heat content. This step ensures the same time-

359 filtering for all aspects of the energy budget, and thereby prevents the introduction of

360 significant errors. In the case of the net TOA imbalance, an energy-leakage constant

361 of $0.44 \mathrm{Wm}^{-2}$ is first estimated from 2000 years of the control run and then removed.

362 Leakage is energy destroyed by model errors; MPI-ESM1.1 has improved energy

363 conservation compared to its predecessor, MPI-ESM, and both have relatively small

364 leakage compared to models in the CMIP5 ensemble ${ }^{34}$.

365 The comparison between flux-divergence and ocean surface-layer heat content

366 (Supplementary Fig. 1) uses a slightly different approach. To test for exact changes in

367 heat content over a 15-year period, only the start and end states are relevant. The

368 least-squares method is however, influenced by the pathway from start- to end-states. 
369 Instead, a difference filter is calculated from the start- and end-years in the 15-year

370 sliding window, divided by the time difference of 14 years: $\Delta X_{t}=\frac{1}{14}\left(X_{t+14}-X_{t}\right)$.

371 The selected flux-divergence is the sum of two components: the TOA radiative

372 imbalance minus atmospheric heat uptake (trends in vertically integrated moist static

373 energy); and trends in ocean heat content below the ocean surface layer. This is the

374 simplest flux combination that matches the expected one-to-one relationship between

375 flux-divergence and change in surface-layer heat content (Supplementary Fig. 1). The

376 salient characteristic of the ocean surface layer for this study is the relationship

377 between heat-content changes within the layer and resulting changes in GMST. The

378 surface-layer depth of $100 \mathrm{~m}$ is therefore chosen to maintain the high correlation

379 between the flux-divergence and GMST trends (Fig. 2b), but remains a conservative

380 choice for estimation of the flux-divergence threshold during hiatuses. Removing heat

381 changes that are related to phase changes (land-ice and sea-ice changes) or including

382 the heat flux from the soil does not improve the relationship with GMST trends

383 (Supplementary Fig. 2).

384 The expected surface-layer flux-divergence associated with a hiatus is calculated from 385 the slope of the regression between flux-divergence and GMST trends. The value we 386 calculate $\left(-0.082 \mathrm{Wm}^{-2}\right)$ is less than the flux-divergence required by uniform cooling 387 of $-0.17^{\circ} \mathrm{C}$ per decade in the top $100 \mathrm{~m}$ of ocean: $-0.150 \mathrm{Wm}^{-2}$. This is because the 388 layer cools on average by only $-0.10^{\circ} \mathrm{C}$ per decade during hiatuses, which matches 389 the theoretically expected cooling if the total anomaly of $-0.082 \mathrm{Wm}^{-2}$ were focussed 390 in the ocean surface layer. The error interval of $\pm 0.038 \mathrm{Wm}^{-2}$ is calculated from the 5$39195 \%$ range of all regression residuals of flux-divergence during hiatuses in the 392 ensemble. There is no significant relationship between the origin of hiatuses and 393 different periods in time (Supplementary Table 2).

394 The heat-content changes for individual basins are calculated from linear trends in 395 heat content below 100m. Basin boundaries are identical to those used in CMIP5 and 396 can be downloaded from the quality-control data in ref. 35.

397 Observational estimates in Figure 3c rely on a combination of data sources, which are 398 summarised below and quantified in Supplementary Table 1. The CERES/WOA 399 estimate for the 2000s is composed from the estimate of TOA fluxes in ref. 21, and an 400 estimate of heat uptake using WOA data ${ }^{22}$, including pentadal heat-content values for 
$401700 \mathrm{~m}-2000 \mathrm{~m}$, yearly heat content values for the upper $700 \mathrm{~m}$, and a separate estimate

402 for deep-ocean warming ${ }^{36}$. From the total heat uptake, we subtract the heat-content

403 trend for the first $100 \mathrm{~m}$ in the WOA objective analysis data ${ }^{22}$ (calculated from in-situ

404 temperature with a constant density and specific heat of $4 \times 10^{6}$ Joules $\mathrm{m}^{-3} \mathrm{C}^{-1}$ ).

405 For this first budget, the 1-sigma error bars for the TOA estimate are taken from the

406 same source as the estimate itself ${ }^{21}$. The error bars for the WOA ocean heat-content

407 trend are calculated as plus or minus the standard error of the slope parameter,

408 assuming that the errors in heat content are auto-correlated and behave like an $\mathrm{AR}(1)$

409 process $^{37,38}$. The auto-correlation coefficient for the errors is estimated from residuals

410 in heat-content data preceding the 2000s (1957-1999). A reduced degrees-of-freedom

411 is calculated from the auto-correlation coefficient and scales the estimate of the

412 standard error in heat content, which is calculated directly from the error estimates

413 provided with the WOA data ${ }^{22}$ (not from the regression residuals).

414 The ORAS4 ocean anomaly is calculated using an estimate for the total-depth heat

415 uptake in the 2000s (ref. 9) minus the trend for the top $100 \mathrm{~m}$, which is calculated

416 from the available ORAS4 potential temperature values with a constant density and

417 specific heat of $4 \times 10^{6}$ Joules $\mathrm{m}^{-3} \mathrm{C}^{-1}$. The 1-sigma error bars are taken directly from

418 ref. 9. For this second budget, the corresponding TOA flux estimate and its error bars

419 are taken from ref. 23.

420 For both observation-based budgets, we remove the effect of ocean drift in the large

421 ensemble. A quadratic function is first fitted to ocean heat content over the 2000 -year

422 control run ${ }^{39}$. Since each ensemble member starts from a different point in the control

423 run, the drift is estimated from the rate-of-change in the quadratic that corresponds to

424 each ensemble member's midpoint. The resulting ensemble-mean drift of $0.01 \mathrm{Wm}^{-2}$

425 is removed from both the ocean component and the TOA component.

426 In ref. 12, the budget is given as anomalies from the control experiment in total heat-

427 content change for the top $125 \mathrm{~m}$ of ocean and the remaining ocean. We convert these

428 values to 15-year fluxes over the total Earth surface. We assume that the anomaly

429 below $125 \mathrm{~m}$ represents the ocean component, and the sum of surface and deep-ocean

430 components is equivalent to the TOA component. 
Code availability. The MPI-ESM1.1 model version was used to generate the large ensemble and is available at http://www.mpimet.mpg.de/en/science/models/mpiesm.html. Computer code used in post-processing of raw data has been deposited with the Max Planck Society:

http://pubman.mpdl.mpg.de/pubman/faces/viewItemFullPage.jsp?itemId=escidoc:235 $\underline{3695}$.

Data availability. Raw data from the large ensemble were generated at the Swiss National Computing Centre (CSCS) and Deutsches Klimarechenzentrum (DKRZ) facilities. Derived data have been deposited with the Max Planck Society (http://pubman.mpdl.mpg.de/pubman/faces/viewItemFullPage.jsp?itemId=escidoc:23 53695). Supplementary Figure 6 uses TOA flux reconstructions provided by R Allan $^{40}$ (http://www.met.reading.ac.uk/ sgs01cll/flux/) and satellite observations provided by the NASA CERES project ${ }^{31}$ (http://ceres.larc.nasa.gov). For observational estimates in Figure $3 \mathrm{c}$, we make use of data provided by the NOAA World Ocean Atlas ${ }^{22}$ (https://www.nodc.noaa.gov/OC5/3M_HEAT_CONTENT/) and by the ECMWF Ocean Reanalysis System 4 (ref. 9; http://icdc.zmaw.de/projekte/easy-init/easy-init-ocean.html).

\section{References}

33. Marotzke, J. \& Forster, P. M. Forcing, feedback and internal variability in global temperature trends. Nature 517, 565-570 (2014).

34. Mauritsen, T. et al. Tuning the climate of a global model. J. Adv. Model. Earth Syst. 4, (2012).

35. Jungclaus, J. et al. CMIP5 simulations of the Max Planck Institute for Meteorology (MPI-M) based on the MPI-ESM-LR model: The decadal2000 experiment, served by ESGF. World Data Center for Climate (WDCC) at DKRZ. http://dx.doi.org/doi:10.1594/WDCC/CMIP5.MXEL00 (2013).

36. Purkey, S. G. \& Johnson, G. C. Warming of global abyssal and deep Southern Ocean waters between the 1990s and 2000s: Contributions to global heat and sea level rise budgets. J. Clim. 23, 6336-6351 (2010).

37. Hartmann, D. L. et al. in Climate Change 2013: The Physical Science Basis. (eds. Stocker, T. F. et al.) 2SM-1-2SM-30 (Cambridge University Press, 2013).

38. Santer, B. D. et al. Consistency of modelled and observed temperature trends in the tropical troposphere. Int. J. Climatol. 28, 1703-1722 (2008).

39. Sen Gupta, A., Jourdain, N. C., Brown, J. N. \& Monselesan, D. Climate drift in the CMIP5 models. J. Clim. 26, 8597-8615 (2013). 
468 40. Allan, R. P. et al. Changes in global net radiative imbalance $1985-2012$. Geophys. Res. Lett. 41, 5588-5598 (2014) 